\title{
ASYMPTOTIC OPTIMALITY OF SEQUENTIAL DESIGNS FOR ESTIMATION
}

\author{
KAMEL REKAB \\ Departanent of Applied Matheindte" \\ Florida Institute of Technology \\ Melbourne, FL 32901 \\ (Received November 5, 1992)
}

\begin{abstract}
This paper is concerned with the problem of allocating a fixed number of trials between $K$ independent populations from the exponential family, in order to estimate a linear combination of the means with squared error loss. Introducing independent conjugate priors, a batch sequential procedure is proposed and compared with the optimal.
\end{abstract}

KEY WORDS AND PHRASES. Bayes rısk; Exponentıal famılıes; Sequential desıgns; Unıform ıntegrabılıty.

1992 AMS SUBJECT CLASSIFICATION CODES. 62L05, 62L10

\section{INTRODUCTION.}

Given $K$ independent populations $P_{1}, \ldots, P_{K}$ indexed by unknown parameters $\alpha_{1}, \ldots, \alpha_{K}$ respectively, it is wished to estimate a linear combination of their respective means based on a fixed total number of observations $M$. The problem is one of allocating the $M$ observations between the $K$ populations so as to minimize expected squared error loss. Several special cases of this problem have been studied.

For estimating the difference in two Binomial means, Alvo and Cabilio. (1982) proposed an allocation procedure shown to be asymptotically optimal. Rekab (1989) considered problem of estimating the product of means of two normal populations. Robbins, Simon, and Starr (1967) considered the problem of estimating the difference of the means of two normal populations with unknown means and unknown variances. They proposed a sampling scheme that has been applied in a variety of sequential estimation problems. Their work was restricted entirely to the ad hoc design. Woodroofe and Hardwick (1990) considered the problem of estimating the difference between the means of two normal populations with ethical costs. Using a quasi Bayesian approach, they proposed a three stage procedure shown to be optimal to second order for squared error loss.

In the present article we adopt a fully Bayesian approach to the problem of estimating a linear combination of the means of $K$ populations from the general exponential family. A lower bound on the Bayes risk is derived and shown to be achieved asymptotically as $M \rightarrow \infty$ by a batch sequential procedure. 


\section{THE BAYESIAN MODEL.}

Let $P_{1} \ldots P_{K}$ be $K$ independent populations from the general exponential fanily indexed by $a$. . . ak respectively: That is. suppose that

$$
d F_{n_{i}}\left(r_{i}\right)=\exp \left\{\left\{\alpha_{i}, r_{i}-\imath \cdot\left(\alpha_{i}\right)\right\} d \lambda\left(x_{i}\right) \quad-\infty<r_{i}<\infty, \quad \alpha_{i} \in \Omega\right.
$$

for $i=1 \ldots K$. It is assumed throughout that $\Omega$ is the natural parameter space of the family and that $\Omega$ is open. It is woll known that $E_{\alpha_{i}}\left(X_{i}\right)=\psi^{\prime}\left(\alpha_{i}\right)$ and $V_{\alpha_{i}}\left(X_{i}\right)=\psi^{\prime \prime}\left(\alpha_{i}\right)$. Let these in turn be assigned independent conjugate priors, that is suppose that $\alpha_{i}$ are independent random variables which have distributions

$$
d \Pi\left(\alpha_{i}\right)=\frac{\exp \left\{r_{i} \mu_{i} \alpha_{i}-r_{i} \psi\left(\alpha_{i}\right)\right\}}{c\left(r_{i}, \mu_{i}\right)} d \alpha_{i}
$$

where

$$
c\left(r_{i}, \mu_{i}\right)=\int \exp \left\{r_{i} \mu_{i} \alpha_{i}-r_{i} \psi\left(\alpha_{i}\right)\right\} d \alpha_{i} .
$$

Let $M$ be the fixed total number of observations and $M_{1}, \ldots, M_{K}$ be the random variables which allocate observations to $P_{1}, \ldots, P_{K}$ with $\sum_{i=1}^{K} M_{i}=M$. In estimating $\sum_{i=1}^{K} c_{i} \psi^{\prime}\left(\alpha_{i}\right)$ with squared error loss, the terminal Bayes estimator is $\sum_{i=1}^{K} c_{i} \mu_{i, M_{i}}$, where $\mu_{i, M_{i}}$ is the mean of the posterior distribution $\psi^{\prime}\left(\alpha_{i}\right)$ based on $M_{i}$ observations. Due to independence of the priors, the terminal Bayes risk $r_{\beta}$ is given by

$$
r_{\beta}=E\left(\sum_{i=1}^{K} \frac{c_{i}^{2} U_{i, M_{i}}}{M_{i}+r_{i}}\right)
$$

where $U_{i, M_{i}}=E\left\{\psi^{\prime \prime}\left(\alpha_{i}\right) \mid X_{i, 1}, \ldots, X_{i, M_{i}}\right\}$.

\section{LOWER BOUND ON THE BAYES RISK.}

We begin this section by deriving a lower bound on the risk incurred by the optimal procedure.

LEMMA 3.1: Let $r_{\beta}$ be defined as in (2.1). Then

$$
r_{\beta} \geq \frac{E\left(\sum_{i=1}^{K}\left(\left|c_{i}\right| \sqrt{\psi^{\prime \prime}\left(\alpha_{i}\right)}\right)^{2}\right)}{M+\sum_{i=1}^{K} r_{i}} .
$$

PROOF: The terminal Bayes risk can be written as the sum of

$$
\left(M+\sum_{i=1}^{K} r_{i}\right)^{-1} E\left(\sum_{i=1}^{K}\left|c_{i}\right| \sqrt{U_{i, M_{i}}}\right)^{2}
$$

and

$$
\left(M+\sum_{i=1}^{K} r_{i}\right)^{-1} E\left\{\sum_{i=1}^{K-1} \sum_{j=i+1}^{K} \frac{\left(\left(M_{i}+r_{i}\right)\left|c_{j}\right| \sqrt{U_{j, M_{j}}}-\left(M_{j}+r_{j}\right)\left|c_{i}\right| \sqrt{U_{i, M_{i}}}\right)^{2}}{\left(M_{i}+r_{i}\right)\left(M_{j}+r_{j}\right)}\right\}
$$

The lemma follows by the martingale properties of $U_{i, M_{i}}$. 


\section{A BATCH SEQUENTIAL PROCEDURE.}

In this section a batch sequential procedure is proposed and shown to be asymptotically optimal. Let $M_{\imath, b}$ denote the number of observations sampled from population $P_{\imath}$ up to stage $b$. Then at stage $b$ the Bayes risk is minimized by

$$
\left(b+\sum_{i=1}^{K} r_{\imath}\right)^{-1} E\left(\sum_{i=1}^{K}\left|c_{\imath}\right| \sqrt{U_{i, M_{i, b}}}\right)^{2}
$$

and achieves its minimum when the following equality is satisfied for all $i$ and $j$

$$
\frac{M_{i, b}+r_{2}}{M_{\jmath, b}+r_{\jmath}}=\frac{\left|c_{\imath}\right| \sqrt{U_{i, M_{i, b}}}}{\left|c_{\jmath}\right| \sqrt{U_{3, M_{, b}}}}
$$

With a motivation to move $\frac{M_{i, b}+r_{i}}{M_{,, b}+r_{r}}$ toward $\frac{\left|c_{i}\right| \sqrt{U_{i, M_{i, b}}}}{\left|c_{j}\right| \sqrt{U_{,, M_{j, b}}}}$, we propose a batch sequential procedure as follows. First start with an initial sample of $K$ observations, one observation from each population. The remaining $M-K$ observations will be allocated in $B$ batches where each batch consists of $K-1$ observations. Suppose up to batch $b$ we already observed $M_{1, b}, \ldots, M_{K, b}$, then select one additional observation from each population excluding population $P_{\mathfrak{z}}$ in next batch if

$$
M_{i, b}+r_{2}>\max _{j \neq i}\left\{\frac{\left(M_{j, b}+r_{j}\right)\left|c_{\imath}\right| \sqrt{U_{i, M_{i, b}}}}{\left|c_{j}\right| \sqrt{U_{j, M_{j, b}}}}\right\}
$$

The following lemmas are needed for the proof of the theorem.

LEMMA 4.1: Using the proposed batch sequential procedure, $M_{\mathbf{z}, b} \rightarrow \infty$ almost surely as $b \rightarrow \infty$ for all $i$.

PROOF: The batch sequential procedure can be written as follows: Sample one observation from each population excluding $P_{i}$ if

$$
\frac{\left(M_{\imath, b}+r_{\imath}\right)\left|c_{j}\right| \sqrt{U_{j, M_{j, b}}}}{\left|c_{\imath}\right| \sqrt{U_{\imath, M_{i, b}}}+\left|c_{j}\right| \sqrt{U_{j, M_{j, b}}}}>\frac{\left(M_{j, b}+r_{\jmath}\right)\left|c_{\imath}\right| \sqrt{U_{\imath, M_{i, b}}}}{\left|c_{\imath}\right| \sqrt{U_{\imath, M_{i, b}}}+\left|c_{j}\right| \sqrt{U_{j, M_{,, b}}}}
$$

for all $j \neq i$. Suppose that $M_{i, b}$ is bounded. Then $M_{\jmath, b}$ are bounded for all $j \neq i$. Hence we have a contradiction.

LEMMA 4.2: For $b$ large enough, let

$$
k^{(\imath)}=\sup \left\{k \leq b: M_{i, k}+r_{i}>\max _{j \neq i}\left\{\frac{\left(M_{j, k}+r_{\jmath}\right)\left|c_{i}\right| \sqrt{U_{i, M_{i, k}}}}{\left|c_{\jmath}\right| \sqrt{U_{j, M_{,, k}}}}\right\}\right.
$$

for all $i=1, \ldots, K$. Then

$$
\begin{aligned}
\frac{\left|c_{\imath}\right| \sqrt{U_{i, M_{i, k}(\cdot)}}}{\left|c_{\jmath}\right| \sqrt{U_{j, M_{j, k}(i)}}}\left(1-\frac{1}{M_{j, k(1)}+r_{\jmath}}\right) & \leq \frac{M_{\imath, b}+r_{i}}{M_{j, b}+r_{j}} \\
& \leq \frac{\left|c_{\imath}\right| \sqrt{U_{\imath, M_{i, k}(\jmath)}}}{\left|c_{\jmath}\right| \sqrt{U_{j, M_{j, k}(\jmath)}}}+\left(\frac{1}{M_{j, k}(\jmath)+r_{\jmath}}\right) .
\end{aligned}
$$


PROOF:

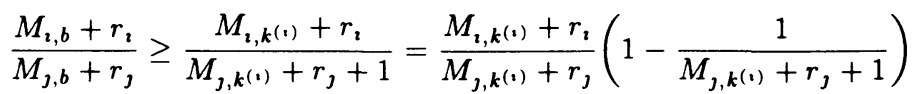

$$
\begin{aligned}
& \geq \frac{\left|c_{2}\right| \sqrt{U_{2, M_{i, k}(1)}}}{\left|c_{\jmath}\right| \sqrt{U_{\jmath, M_{J, k}(1)}}}\left(1-\frac{1}{M_{J, k^{(1)}}+r_{\jmath}}\right) \text {. }
\end{aligned}
$$

On the other hand,

$$
\begin{aligned}
\frac{M_{\imath, b}+r_{2}}{M_{\jmath, b}+r_{\jmath}} & \leq \frac{M_{\imath, k}(\jmath)+r_{\imath}+1}{M_{\jmath, k(\jmath)}+r_{\jmath}}=\frac{M_{\imath, k}(\jmath)+r_{2}}{M_{\jmath, k(\jmath)}+r_{\jmath}}+\frac{1}{M_{\jmath, k}(\jmath)+r_{\jmath}} \\
& \leq \frac{\left|c_{\imath}\right| \sqrt{U_{\imath, M_{\imath, k}(\jmath)}}}{\left|c_{\jmath}\right| \sqrt{U_{\jmath, M_{\jmath, k}(\jmath)}}}+\frac{1}{M_{\jmath, k}(\jmath)+r_{\jmath}} .
\end{aligned}
$$

THEOREM 4.1: Let $r_{\mathcal{P}}$ be the risk incurred by the proposed batch sequential procedure. Suppose there exists $p>1$ such that $E\left(\psi^{\prime \prime}\left(\alpha_{\imath}\right)\right)^{p}<\infty$ for all $i=1, \ldots, K$.

$$
r_{\mathcal{P}}=\frac{E\left(\sum_{\imath=1}^{K}\left(\left|c_{\imath}\right| \sqrt{\psi^{\prime \prime}\left(\alpha_{\imath}\right)}\right)^{2}\right)}{M+\sum_{\imath=1}^{K} r_{\imath}}+o(1 / M)
$$

as $M \rightarrow \infty$.

PROOF: The proof of the theorem will follow if we establish

$$
E\left(\sum_{i=1}^{K}\left|c_{\imath}\right| \sqrt{U_{i, M_{i}}}\right)^{2}-E\left(\sum_{i=1}^{K}\left|c_{\imath}\right| \sqrt{\psi^{\prime \prime}\left(\alpha_{\imath}\right)}\right)^{2} \rightarrow 0 \quad \text { as } \quad M \rightarrow \infty
$$

and

$$
E\left\{\sum_{\imath=1}^{K-1} \sum_{\jmath=\imath+1}^{K} \frac{\left(\left(M_{\imath}+r_{\imath}\right)\left|c_{\jmath}\right| \sqrt{U_{,}, M_{\jmath}}-\left(M_{\jmath}+r_{\jmath}\right)\left|c_{\imath}\right| \sqrt{U_{\imath}, M_{\imath}}\right)^{2}}{\left(M_{\imath}+r_{\imath}\right)\left(M_{\jmath}+r_{\jmath}\right)}\right\} \rightarrow 0
$$

as $M \rightarrow \infty$. Let $M_{1}, \ldots, M_{K}$ be the allocation variables for the batch sequential procedure. Since $M_{\imath} \rightarrow \infty$ and $U_{\imath, M}$, are uniformly integrable, (4.1) is satisfied. Using Lemma (4.2),

$$
\frac{M_{\imath}}{M_{3}} \rightarrow \frac{\left|c_{\imath}\right| \sqrt{\psi^{\prime \prime}\left(\alpha_{\imath}\right)}}{\left|c_{2}\right| \sqrt{\psi^{\prime \prime}\left(\alpha_{\jmath}\right)}} \quad \text { a.s. } \quad \text { as } M \rightarrow \infty .
$$

The proof will be established if $\frac{M_{1}+r_{1}}{M_{\jmath}+r_{\jmath}}\left(c_{\jmath}\right)^{2} U_{\jmath, M}$, is uniformly integrable. Using Lemma (4.2),

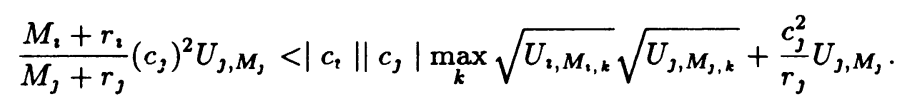

The proof follows by Doob's inequality for non negative submartingales.

\section{REFERENCES}

[1] Alvo, M. and Cabilio, P. (1982). Bayesian estimation of the difference between two proportions. Canadian J. Statist. 10, 139-145.

[2] Rekab, K. (1989). Asymptotic efficiency in sequential designs for estimation. Sequential Analysis 8, 269-280.

[3] Robbins, H., Simons, G., and Starr, N. (1967). A sequential analogue of the Behren-Fisher problem. Ann. Math. Stat 38, 1384-1388.

[4] Woodroofe, M., Hardwick, J. (1990). Sequential allocation for estimation problem with ethical costs. Ann. Stat 18, 1358-1377. 


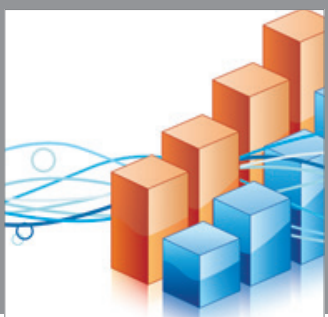

Advances in

Operations Research

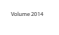

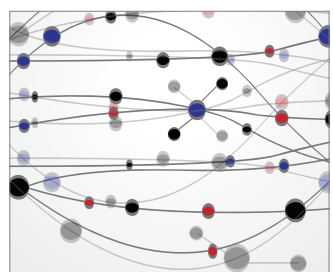

\section{The Scientific} World Journal
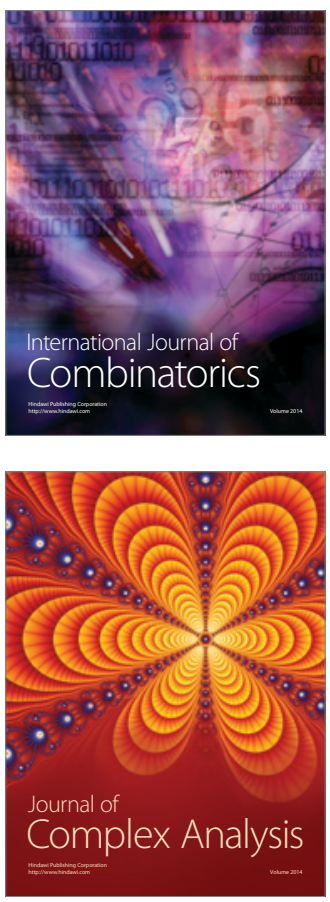

International Journal of

Mathematics and

Mathematical

Sciences
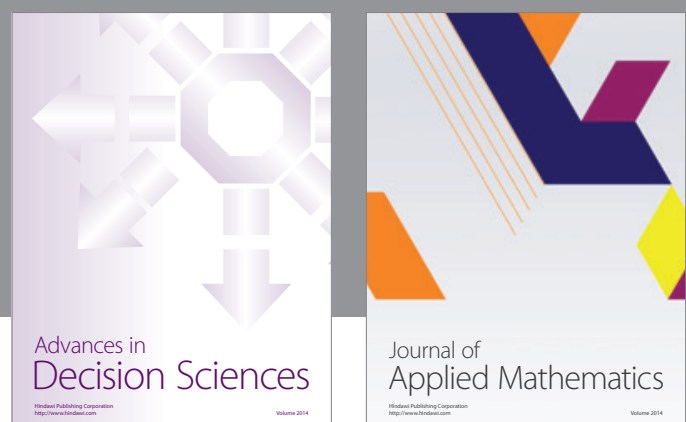

Journal of

Applied Mathematics
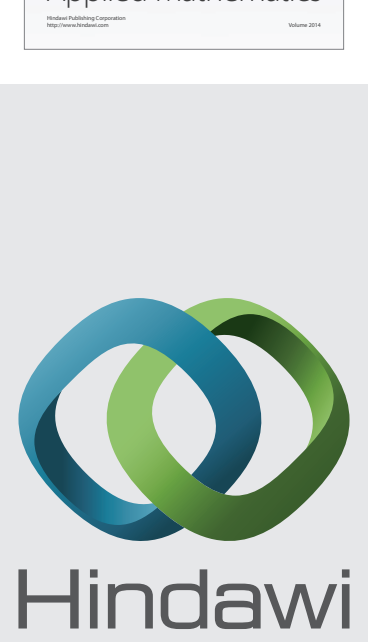

Submit your manuscripts at http://www.hindawi.com
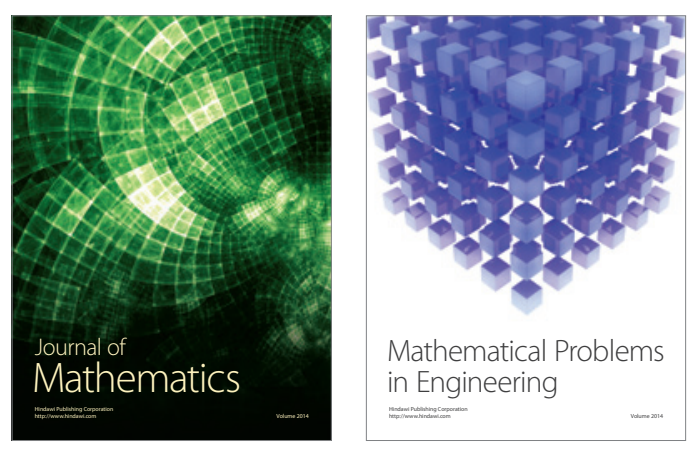

Mathematical Problems in Engineering
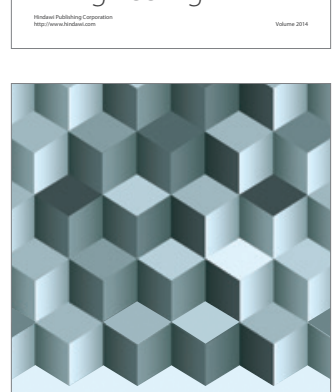

Journal of

Function Spaces
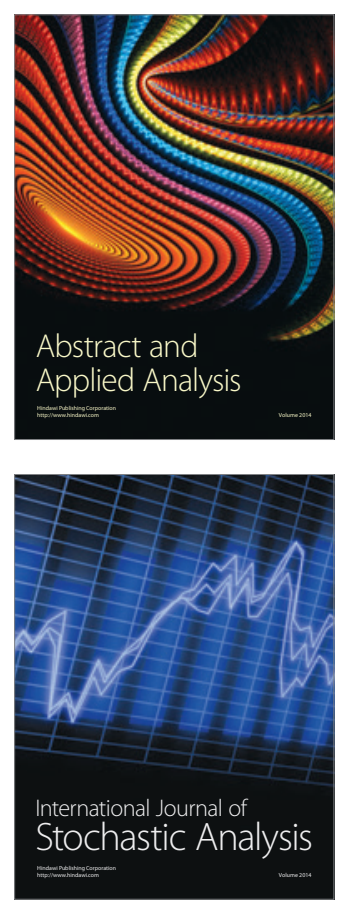

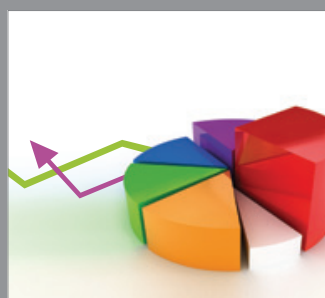

ournal of

Probability and Statistics

Promensencen
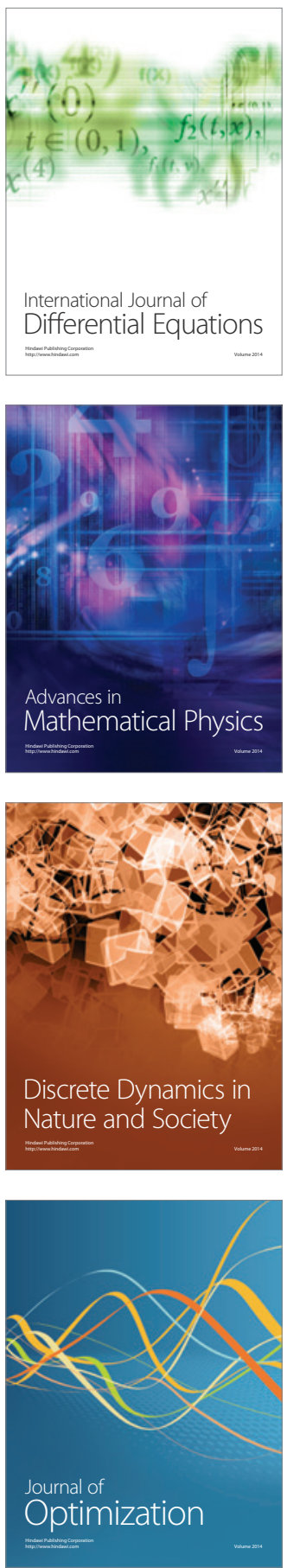